\title{
Mode-resolved Cavity-enhanced Vernier Spectroscopy Using an Interband Cascade Laser Frequency Comb
}

\author{
Lukasz A. Sterczewski ${ }^{1}$, Tzu-Ling Chen ${ }^{2}$, Douglas C. Ober ${ }^{2}$, Charles R. Markus ${ }^{2}$, Chadwick L. Canedy ${ }^{3}$, \\ Igor Vurgaftman ${ }^{3}$, Clifford Frez ${ }^{1}$, Jerry R. Meyer ${ }^{3}$, Mitchio Okumura ${ }^{2}$, and Mahmood Bagheri ${ }^{1}$ \\ ${ }^{1}$ Jet Propulsion Laboratory, California Institute of Technology, Pasadena, California 91109, USA \\ ${ }^{2}$ Division of Chemistry and Chemical Engineering, California Institute of Technology, Pasadena, CA, 91125, USA \\ ${ }_{3}^{3}$ Code, 5613, Naval Research Laboratory, Washington, DC 20375, USA \\ Author e-mail address: lukasz.a.sterczewski@jpl.nasa.gov
}

\begin{abstract}
We demonstrate a mid-IR mode-resolved Vernier optical frequency comb spectrometer with an interband cascade laser source. The free-running system provides 35 meters of effective path length for monitoring 1-THz-broad spectra at $3.63 \mu \mathrm{m}$. $\odot 2021$ The Author(s)
\end{abstract}

\section{Introduction}

Chip-scale optical frequency combs (OFCs) [1] are revolutionizing the detection of trace chemicals in gas form, by providing high-resolution, broadband spectroscopy at a rapid sampling speed. Currently, dual-comb spectroscopy (DCS) [2] is arguably the most widely-used approach for accessing mode-resolved spectral information. Despite its many advantages, however, DCS can be challenging experimentally because high mutual coherence between the two comb lasers must be maintained via analog [3] or digital [4] feedback control loops, and precise broadband microwave digitizing is needed. Moreover, high sensitivity requires enhancement of the interaction length via a multi-pass Herriott cell or optical cavity that adds an extra layer of complexity to the OFC spectrometer.

In this work, we demonstrate a simplified Vernier spectroscopy approach [5] that provides robust, real-time sensing. As illustrated in Fig. 1, we employ a high-finesse $(F=1800)$ optical cavity in combination with a single-pixel photodetector to simultaneously realize both optical path enhancement and the full spectral resolution of a single free-running interband cascade laser (ICL) OFC.

\section{Vernier spectrometer}
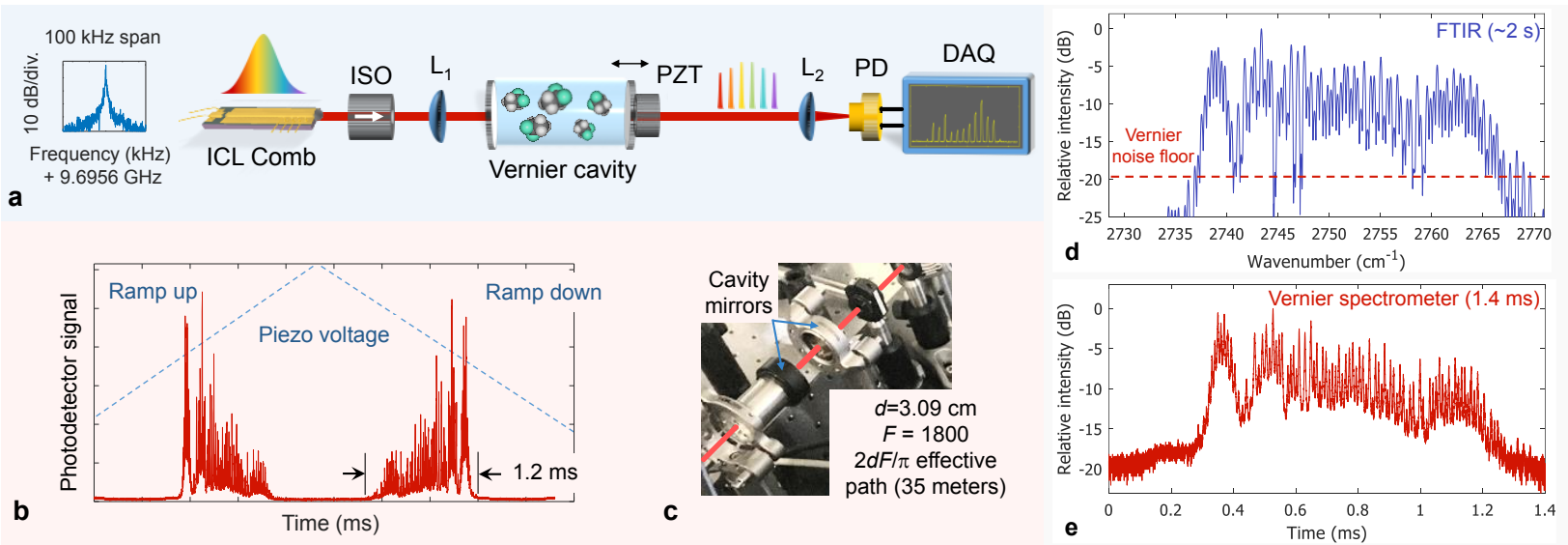

Fig. 1. Vernier OFC spectrometer. (a) Schematic of the experimental setup. Collimated light from a freerunning ICL comb is mode-matched to the cavity using a lens (L1) preceded by an optical isolator (ISO). Voltage tuning of the piezoelectric transducer (PZT) enables the cavity to act as a mode-selecting optical filter. A single-element photodetector (PD) converts light into an electrical signal that is captured by a digitizer (DAQ). (b) Example of a time-domain trace acquired with a $7 \mathrm{MS} / \mathrm{s}$ 12-bit digitizer. (c) Photograph of the open-path Vernier cavity with effective path length $35 \mathrm{~m}$. (d) Optical spectrum measured with a Fourier Transform spectrometer (FTIR) in $\sim 2$ seconds. (e) Optical spectrum measured in $1.4 \mathrm{~ms}$ by scanning the mirror spacing of the Vernier cavity. Because the optical linewidth of $\sim 300 \mathrm{kHz}$ at $1 \mathrm{~ms}$ increases slightly with frequency due to the accumulation of timing jitter, a lower coupling efficiency around $2765 \mathrm{~cm}^{-1} \mathrm{causes}^{-1}$ the comb lines to have amplitudes $\sim 1 \mathrm{~dB}$ lower than those at $2737 \mathrm{~cm}^{-1}$. Nevertheless, the full comb bandwidth is transmitted through the cavity. 
The Vernier approach is a direct-frequency-comb spectroscopy method [5] that intentionally mismatches the optical cavity's free spectral range (FSR) with respect to the comb repetition rate $f_{\text {rep. }}$. A millisecond-long voltage ramp applied to a piezoelectric transducer (PZT) provides scanning of the mirror spacing and hence the FSR, so as to periodically align the resonance with one comb tooth at a time. Consequently, the cavity acts as a tunable, highresolution optical filter. The ultra-compact footprint of the ICL comb source [6], and the small size of the cavity itself make this a promising instrument for spectroscopic sensing that is fully compatible with free-running comb operation. Any drift of the unstabilized comb's optical frequencies causes merely a change in the time of arrival for individual lines, which can easily be corrected by a digital signal processing algorithm similar to those used in DCS [4]. The primary difference is that the lines in the scan are not equidistant, which occurs because over a submillisecond timescale, strong optical feedback from the non-resonant cavity affects the comb tooth lasing frequency, even with an optical isolator (ISO) in the beam path. Furthermore, minor thermal fluctuations are likely to affect the lasing frequency over longer time spans of seconds to minutes. Figure 2 shows that semi-coherent averaging is possible over second-long timescales via digital resampling of the spectrum onto a uniform grid, followed by alignment of the $f_{\text {rep }}$-corrected spectra. Note also that the single-shot $(2 \mathrm{~ms})$ amplitude precision of the most intense lines is $\approx 1 \%$, i.e., already sufficiently low for accurate monitoring of many transient events. The Allan-Werle deviation of the peak intensity reaches a minimum of $1.26 \times 10^{-3}$ at $240 \mathrm{~ms}$, followed by a gradual drift at longer timescales. This corresponds to a minimum detectable absorption (MDA) of $6.17 \times 10^{-4} \mathrm{~Hz}^{-1 / 2}$ and a noise-equivalent absorption sensitivity (NEAS) of $1.76 \times 10^{-7} \mathrm{~cm}^{-1} \mathrm{~Hz}^{-1 / 2}$. Surprisingly, this is only an order of magnitude less sensitive than is attainable from a large-size, quasi-stabilized OPO-based Vernier system [7] operating in the mid-infrared.
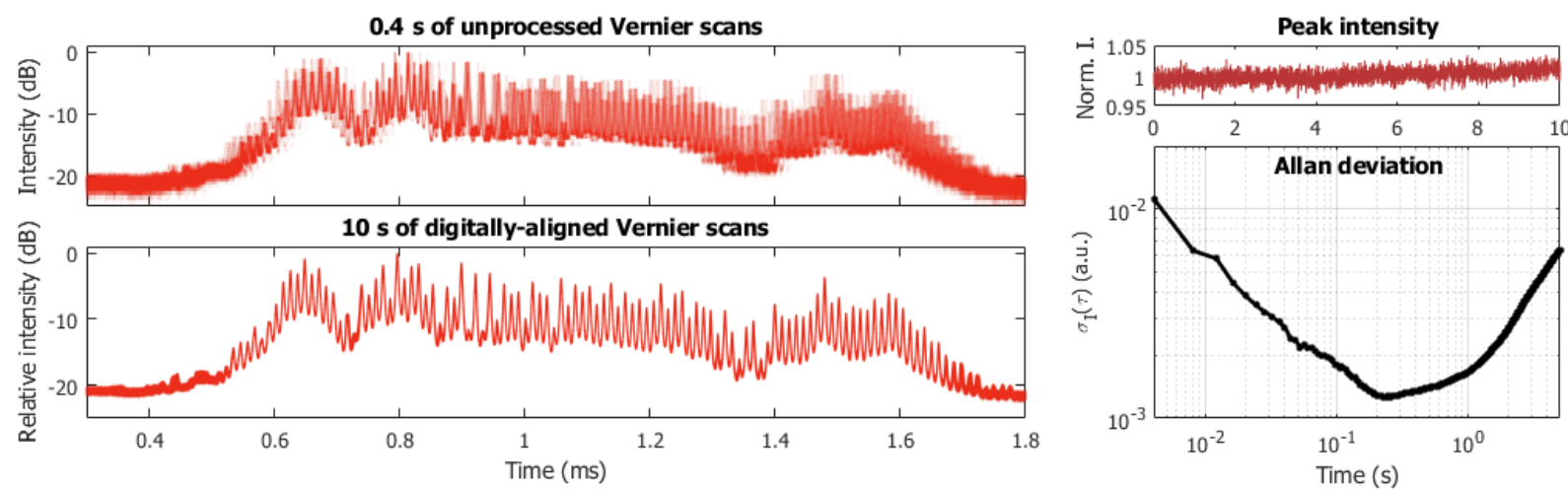

Fig. 2. Digital post-correction of scans from the mode-resolved Vernier spectrometer. Timing jitter causes the higher-frequency lines (between 1.2 and $1.6 \mathrm{~ms}$ ) to fluctuate more than those at lower frequencies. The correction algorithm allows an entire 10-s acquisition to be aligned for semi-coherent averaging and easy analysis. The Allan-Werle deviation and time series of the strongest line intensity are plotted on the right.

\section{Conclusion}

We have demonstrated an ultra-simple mid-infrared mode-resolved Vernier spectrometer that uses a free-running chip-scale interband cascade laser frequency comb source. The system can measure 1-THz-broad spectra at $\sim 3.63 \mu \mathrm{m}$, with millisecond temporal resolution and permille precision at sub-second timescales. The enhanced interaction length of 35 meters, provided by a $3 \mathrm{~cm}$ long optical cavity, will provide sensitive open-path detection of many environmentally-important molecular species, including spectrally-broadband hydrocarbons.

Acknowledgement: This work was supported under and was in part performed at the Jet Propulsion Laboratory (JPL), California Institute of Technology, under contract with NASA (PICASSO program 106822/811073.02.24.01.85; PDRDF program). L. A. Sterczewski's research was supported by an appointment to the NASA Postdoctoral Program at JPL, administered by Universities Space Research Association under contract with NASA. The NRL authors acknowledge support from the Office of Naval Research (ONR).

\section{References}

1. G. Scalari, J. Faist, and N. Picqué, "On-chip mid-infrared and THz frequency combs for spectroscopy," Applied Physics Letters 114, 150401 (2019).

2. $\quad$ I. Coddington, N. Newbury, and W. Swann, "Dual-comb spectroscopy," Optica 3, 414 (2016).

3. E. Baumann, F. R. Giorgetta, W. C. Swann, A. M. Zolot, I. Coddington, and N. R. Newbury, "Spectroscopy of the methane v3 band with an accurate midinfrared coherent dual-comb spectrometer," Phys. Rev. A 84, 062513 (2011).

4. L. A. Sterczewski, J. Westberg, and G. Wysocki, "Computational coherent averaging for free-running dual-comb spectroscopy," Opt. Express, OE 27, 23875-23893 (2019).

5. C. Gohle, B. Stein, A. Schliesser, T. Udem, and T. W. Hänsch, "Frequency Comb Vernier Spectroscopy for Broadband, High-Resolution, HighSensitivity Absorption and Dispersion Spectra," Phys. Rev. Lett. 99, 263902 (2007).

6. M. Bagheri, C. Frez, L. A. Sterczewski, I. Gruidin, M. Fradet, I. Vurgaftman, C. L. Canedy, W. W. Bewley, C. D. Merritt, C. S. Kim, M. Kim, and J. R. Meyer, "Passively mode-locked interband cascade optical frequency combs," Scientific Reports 8, 3322 (2018).

7. A. Khodabakhsh, L. Rutkowski, J. Morville, and A. Foltynowicz, "Mid-infrared continuous-filtering Vernier spectroscopy using a doubly resonant optical parametric oscillator," Appl. Phys. B 123, 210 (2017). 